\title{
The Rainbow Connection Number of Triangular Snake Graphs
}

\author{
Aleffer Rocha ${ }^{1}$, Sheila M. Almeida ${ }^{1}$, Leandro M. Zatesko ${ }^{2}$ \\ ${ }^{1}$ Academic Department of Informatics \\ Federal University of Technology - Paraná (UTFPR), Ponta Grossa, Brazil \\ ${ }^{2}$ Academic Department of Informatics \\ Federal University of Technology - Paraná (UTFPR), Curitiba, Brazil \\ aleffer@alunos.utfpr.edu.br, \{sheilaalmeida, zatesko\}@utfpr.edu.br
}

\begin{abstract}
Rainbow coloring problems, of noteworthy applications in Information Security, have been receiving much attention last years in Combinatorics. The rainbow connection number of a graph $G$ is the least number of colors for a (not necessarily proper) edge coloring of $G$ such that between any pair of vertices there is a path whose edge colors are all distinct. In this paper we determine the rainbow connection number of the triple triangular snake graphs.
\end{abstract}

\section{Introduction}

We address the problem of determining the rainbow connection number of a graph, introduced more than 10 years ago [Chartrand et al. 2008]. Rainbow colorings appear in many different contexts of Combinatorics [Ehard et al. 2019, Montgomery et al. 2019]. Let $k$ be a non-negative integer and $c: E(G) \rightarrow\{0,1, \ldots, k-1\}$ a not necessarily proper edge-coloring of a graph $G$. A path $P$ in $G$ is rainbow if the colors of the edges in $P$ are pairwise distinct. The edge-coloring $c$ is a rainbow coloring if for every pair of vertices there is a rainbow path connecting them [Chartrand et al. 2008]. The rainbow connection number of $G$, denoted $r c(G)$, is the least number of colors for which there is a rainbow coloring of $G$. Rainbow connectivity has been receiving attention in the last years, being some works funded by the National Security Agency (NSA) of the United States [Lo 2012, Dudek et al. 2015], due to the applications on secure transferring of classified information after the terrorist attacks of September 11, 2001 [Li and Sun 2012].

In this paper, every graph is simple, connected, and non-trivial. Given a graph $G$, determining if $r c(G) \leq k$, for any fixed $k \geq 2$, is an NP-complete problem [Chakraborty et al. 2011, Ananth et al. 2011, Li and Li 2011]. In view of this hardness, several authors engaged efforts in the searching for upper and lower bounds for the rainbow connection number [Chakraborty et al. 2011, Chandran and Rajendraprasad 2012, Schiermeyer 2009]. Despite such effort, the problem is completely solved only for specific and structured graph classes, such as complete graphs, paths, and cycles [Chartrand et al. 2008].

In this paper we determine the rainbow connection number of the triple triangular snake graphs ${ }^{1}$, a class commonly studied in labeling problems [Gallian 2014, Sandhya et al. 2016]. To the best of our knowledge, this parameter is unknown for this graphs, regardless of some investigation ${ }^{2}$.

\footnotetext{
${ }^{1}$ In Portuguese, cobras triangulares triplas.

${ }^{2}$ Rainbow connectivity has been considered for triangular snake graphs in a paper by D. Parmar, (cont.)
} 


\section{Preliminaries}

The distance between two vertices $u$ and $v$, denoted $\operatorname{dist}(u, v)$, of a graph $G$ is the number of edges in a shortest path between $u$ and $v$ in $G$. The diameter of $G$, denoted $\operatorname{diam}(G)$, is the largest distance between a pair of vertices of $G$. Observe that, in a rainbow coloring of $G$, between two vertices $u$ and $v$ such that $\operatorname{dist}(u, v)=\operatorname{diam}(G)$ there must be a path requiring at least $\operatorname{diam}(G)$ colors. This implies $\operatorname{rc}(G) \geq \operatorname{diam}(G)$ for any graph $G$. Moreover, $r c(G) \leq|V(G)|-1$, since a rainbow coloring of $G$ can be obtained assigning to the edges of a spanning tree of $G$ colors which are all distinct [Chartrand et al. 2008].

Among the earliest results on rainbow coloring graph classes are: $\operatorname{rc}\left(T_{n}\right)=n-1$ for any tree $T_{n}$ on $n$ vertices; $r c\left(K_{n}\right)=1$ for the complete graph on $n$ vertices; $r c\left(C_{n}\right)=$ $\lceil n / 2\rceil$ for the cycle $C_{n}$ on $n$ vertices [Chartrand et al. 2008]. Also, rainbow coloring has been receiving attention in the context of graph operations, specially the Cartesian product of graphs [Li and Sun 2010, Rocha and Almeida 2017].

Let $\ell$ and $n$ be positive integers and $n \geq 2$. The $\ell$-triangular snake graph, denoted $T S_{n}^{\ell}$, is defined by $V\left(T S_{n}^{\ell}\right)=\left\{v_{i}: 0 \leq i<n\right\} \cup\left\{v_{i, i+1}^{k}: 0 \leq i \leq n-2 \wedge 0 \leq k<\ell\right\}$ and $E\left(T S_{n}^{\ell}\right)=\left\{v_{i} v_{i+1}: 0 \leq i \leq n-2\right\} \cup\left\{v_{i} v_{i, i+1}^{k}: 0 \leq i \leq n-2 \wedge 0 \leq k<\ell\right\} \cup\left\{v_{i, i+1}^{k} v_{i+1}:\right.$ $0 \leq i \leq n-2 \wedge 0 \leq k<\ell\}$. If $\ell=1$, the graph is simply called a triangular snake. If $\ell=2$, it is called a double triangular snake. If $\ell=3$, it is a triple triangular snake (see figures in the next section). The diameter of $T S_{n}^{\ell}$ is the distance between a vertex of $\left\{v_{0}\right\} \cup\left\{v_{0,1}^{k}: 0 \leq k<\ell\right\}$ and a vertex of $\left\{v_{n-1}\right\} \cup\left\{v_{n-2, n-1}^{k}: 0 \leq k<\ell\right\}$. When $n=2$ and $\ell \geq 2$, the distance between $v_{0}$ and $v_{n-1}$ is 2 . When $n>2$, the distance between $v_{0}$ and $v_{n-1}$ is $n-1$. So, if $n=2$ and $\ell \geq 2$, then $\operatorname{diam}\left(T S_{n}^{\ell}\right)=2$; otherwise, $\operatorname{diam}\left(T S_{n}^{\ell}\right)=n-1$.

\section{The result}

We prove the following.

Theorem 1. Let $G$ be a triple triangular snake graph $T S_{n}^{3}$.

$$
r c(G)= \begin{cases}n, & \text { if } n=2 \text { or } n=3 \\ n-1, & \text { if } n>3 .\end{cases}
$$

Proof. Consider $n=2$. It is sufficient to present a rainbow coloring for the graph $G$ using $\operatorname{diam}(G)=2$ colors. Assign the color 1 for the edges $v_{0} v_{0,1}^{0}$ and $v_{0,1}^{2} v_{1}$. Assign the color 0 for the remaining edges. Clearly, this coloring is a rainbow coloring of $G$.

Consider $n=3$. We shall first show that it is possible to construct a rainbow coloring for $G$ using $\operatorname{diam}(G)+1=3$ colors. For all $k \in\{0,1,2\}$, assign the color 0 for the edges $v_{0} v_{1}$ and $v_{0,1}^{k} v_{1}$, the color 1 for $v_{1} v_{2}$ and $v_{1} v_{1,2}^{k}$, and the color $k$ for $v_{0} v_{0,1}^{k}$ and $v_{1,2}^{k} v_{2}$. The rainbowness of this coloring is again straightforward to verify.

Still in the case $n=3$, assume for the sake of contradiction that $G$ has a rainbow coloring with only 2 colors. Let $X_{i}=\left\{v_{i}, v_{i+1}\right\} \cup\left\{v_{i, i+1}^{k}: 0 \leq k<3\right\}$ for $i \in\{0,1\}$.

(cont.) P. V. Shah and B. Suthar of 2019, entitled Rainbow Connection Number of Triangular Snake Graph, in a predatory journal. The authors correctly verify that the rainbow connection number of triangular snakes and double triangular snakes is equal to its diameter. For triple triangular snakes, we have found both the statement and the proof of the results wrong (the value claimed for the rainbow connection number could be greater than the diameter even for $n \geq 3$ ). 
Without loss of generality, let 0 be the color of $v_{0} v_{1}$. Since there is a rainbow path between $v_{0}$ and $x$ for all $x \in X_{1} \backslash X_{0}$, and since the 0 -colored edge $v_{0} v_{1}$ is the first edge in these paths, the edges $v_{1} v_{2}$ and $v_{1} v_{1,2}^{k}$ for $0 \leq k<3$ must be colored 1 (see Fig. 1(a)). This scenario leaves each among $v_{1,2}^{0} v_{2} v_{1,2}^{1}$ and $v_{1,2}^{0} v_{2} v_{1,2}^{2}$ as the only possibility for a rainbow path of length 2 between $v_{1,2}^{0}$ and each among $v_{1,2}^{1}$ and $v_{1,2}^{2}$, respectively. Since both paths share the edge $v_{1,2}^{0} v_{2}$, we must have $v_{2} v_{1,2}^{1}$ and $v_{2} v_{1,2}^{2}$ colored the same (see Fig. 1(b) for the case wherein $v_{1,2}^{0} v_{2}$ is colored 1 ). However, the only paths of length 2 between $v_{1,2}^{1}$ and $v_{1,2}^{2}$ are $v_{1,2}^{1} v_{1} v_{1,2}^{2}$ and $v_{1,2}^{1} v_{2} v_{1,2}^{2}$, which are both monochromatic, a contradiction.

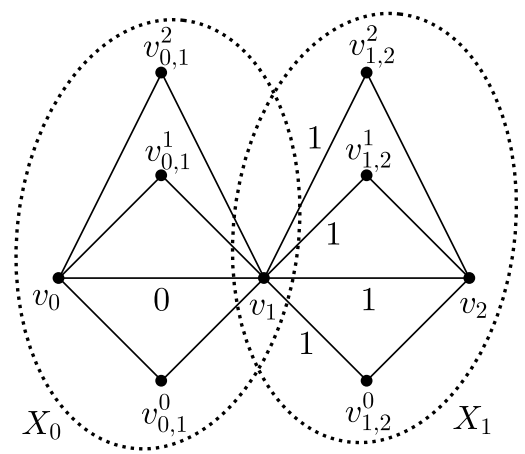

$(a)$

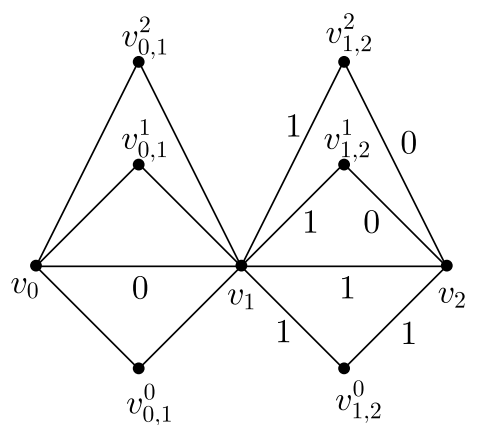

(b)

Figure 1. Constructing a rainbow coloring of $\mathrm{TS}_{3}^{3}$

At last, consider $n>3$. We shall construct a rainbow coloring for $G$ using $\operatorname{diam}(G)=n-1$ colors. For $0 \leq i \leq n-2$, the $i^{\text {th }}$ block is defined as the subgraph induced by the edges $v_{i} v_{i+1}, v_{i} v_{i, i+1}^{k}, v_{i, i+1}^{k} v_{i+1}$ for all $k \in\{0,1,2\}$. The coloring of the first three blocks $(0 \leq i \leq 2)$ is shown in Fig. 2 .

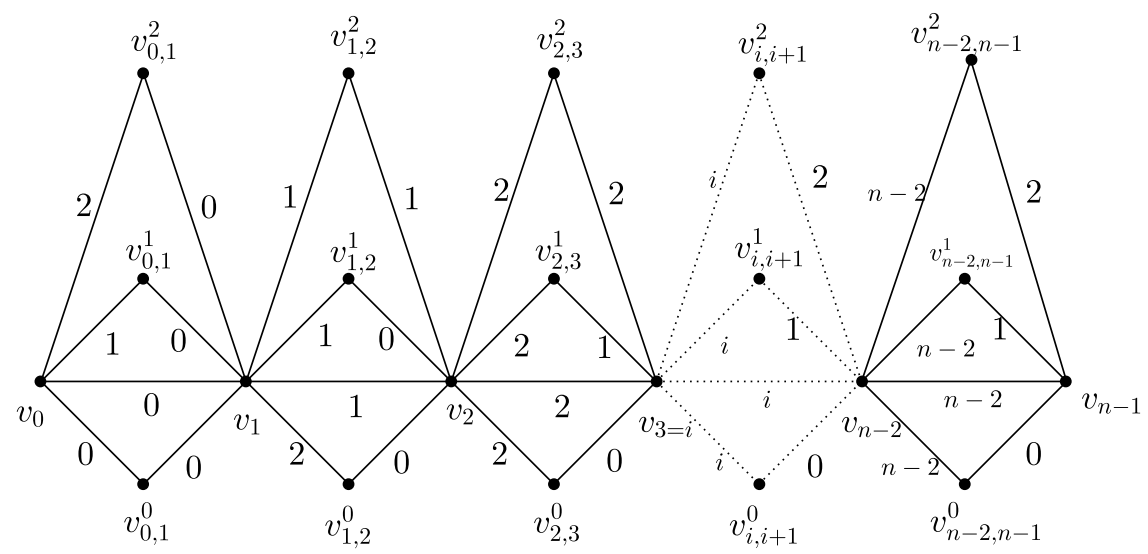

Figure 2. The rainbow coloring of the graph $\operatorname{TS}_{\mathrm{n}}^{3}$ with $\mathrm{n}>3$

For $i>2$, the coloring of the $i^{\text {th }}$ block generalizes the coloring for $i=2$ in the following way: for $0 \leq k \leq 2$, the edges $v_{i} v_{i+1}$ and $v_{i} v_{i, i+1}^{k}$ are colored $i$, and the edges $v_{i, i+1} v_{i+1}^{k}$ are colored $k$ (see again Fig. 2).

Now we verify that under the coloring shown there is a rainbow path between any pair of vertices $u$ and $v$. If $u=v_{i, i+1}^{k^{\prime}}$ and $v=v_{i, i+1}^{k^{\prime \prime}}$ are in the same $i^{\text {th }}$ block for 
$0 \leq i \leq n-2$ and $\left\{k^{\prime}, k^{\prime \prime}\right\} \subseteq\{0,1,2\}$, then the rainbow path between them is the path of length 2 which passes through $v_{i+1}$. As it can also be verified in Fig. 2, in any other case there is only one shortest path between $u$ and $v$, and this path is rainbow.

\section{Acknowledgements}

We thank the reviewers for the valuable suggestions given. This work has been partially supported by CNPq (grant 428941/2016-8) and UTFPR.

\section{References}

Ananth, P., Nasre, M., and Sarpatwar, K. K. (2011). Rainbow connectivity: Hardness and tractability. In IARCS Annual Conference on Foundations of Software Technology and Theoretical Computer Science (FSTTCS 2011), volume 13 of Leibniz International Proceedings in Informatics (LIPIcs), pages 241-251, Dagstuhl, Germany.

Chakraborty, S., Fischer, E., Matsliah, A., and Yuster, R. (2011). Hardness and algorithms for rainbow connectivity. Journal of Combinatorial Optimization, 21:330-347.

Chandran, L. S. and Rajendraprasad, D. (2012). Rainbow colouring of split and threshold graphs. In Int. Computing and Combinatorics Conference, pages 181-192. Springer.

Chartrand, G., Johns, G. L., McKeon, K. A., and Zhang, P. (2008). Rainbow connection in graphs. Mathematica Bohemica, 133(1):85-98.

Dudek, A., Frieze, A. M., and Tsourakakis, C. E. (2015). Rainbow connection of random regular graphs. SIAM Journal on Discrete Mathematics, 29(4):2255-2266.

Ehard, S., Glock, S., and Joos, F. (2019). A rainbow blow-up lemma for almost optimally bounded edge-colourings. arXiv preprint arXiv:1907.09950.

Gallian, J. A. (2014). A dynamic survey of graph labeling. The Electronic journal of combinatorics, 17:60-62.

$\mathrm{Li}, \mathrm{S}$. and Li, X. (2011). Note on the complexity of deciding the rainbow connectedness for bipartite graphs. arXiv preprint arXiv:1109.5534.

Li, X. and Sun, Y. (2010). Characterize graphs with rainbow connection number $m-2$ and rainbow connection numbers of some graph operations. Preprint.

Li, X. and Sun, Y. (2012). Rainbow connections of graphs. Springer.

Lo, I. Y. (2012). Some bounds on the rainbow connection number of 3, 4 and 5-connected graphs. Preprint arXiv:1212.5934.

Montgomery, R., Pokrovskiy, A., and Sudakov, B. (2019). Decompositions into spanning rainbow structures. Proceedings of the London Mathematical Society, 119(4):899-959.

Rocha, A. and Almeida, S. M. (2017). Coloração arco-íris em grafos resultantes de produto cartesiano. In Proc. 37th Congress of the Brazilian Computer Society (CSBC '17/II ETC), pages 83-86, São Paulo.

Sandhya, S., Merly, E. E. R., and Deepa, S. (2016). Heronian mean labeling of triple triangular and triple quadrilateral snake graphs. International Journal of Applied Mathematical Sciences, 9:177-186.

Schiermeyer, I. (2009). Rainbow connection in graphs with minimum degree three. In International Workshop on Combinatorial Algorithms, pages 432-437. Springer. 